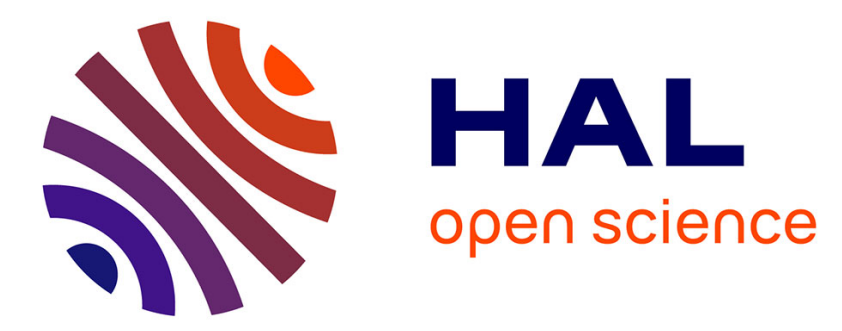

\title{
Influence des liquides hydrophiles sur la gélatine des émulsions photographiques utilisées en physique nucléaire
}

\author{
L. Vigneron, J. Génin, R. Chastel
}

\section{To cite this version:}

L. Vigneron, J. Génin, R. Chastel. Influence des liquides hydrophiles sur la gélatine des émulsions photographiques utilisées en physique nucléaire. J. Phys. Radium, 1955, 16 (3), pp.179-182. 10.1051/jphysrad:01955001603017900 . jpa-00235123

HAL Id: jpa-00235123

https://hal.science/jpa-00235123

Submitted on 1 Jan 1955

HAL is a multi-disciplinary open access archive for the deposit and dissemination of scientific research documents, whether they are published or not. The documents may come from teaching and research institutions in France or abroad, or from public or private research centers.
L'archive ouverte pluridisciplinaire HAL, est destinée au dépôt et à la diffusion de documents scientifiques de niveau recherche, publiés ou non, émanant des établissements d'enseignement et de recherche français ou étrangers, des laboratoires publics ou privés. 


\title{
INFLUENCE DES LIQUIDES HYDROPHILES SUR LA GÉLATINE DES ÉMULSIONS PHOTOGRAPHIQUES UTILISÉES EN PHYSIQUE NUGLÉAIRE
}

\author{
Par L. VIGNERON, Mme J. GÉNIN et R. CHASTEL, \\ Laboratoire de Physique et Chimie nucléaires du Collège de France.
}

\begin{abstract}
Sommaire. - Des émulsions nucléaires imprégnées d'eau ont été séchées à l'air, par le vide ou par immersion dans un liquide hydrophile tel que l'acétone. Dans ces dernières plaques, on constate que les dimensions des traces de particules $\alpha$ subissent des perturbations importantes qui s'interprètent si l'on admet qu'une émulsion photographique quelconque déshydratée par un liquide prend une structure poreuse. Cette structure poreuse reste' stable à sec, elle est détruite si l'on fait gonfler la gélatine dans l'eau. Par ailleurs, l'existence des pores a été confirmée par des expériences de pesée.
\end{abstract}

Pour faire pénétrer des ions, radioactifs ou non, dans une émulsion nucléaire, on opère souvent en immergeant celle-ci dans une solution ionique convenable. Ensuite, il faut sécher cette émulsion humide, tout en conservant intégralement ses propriétés photographiques.

Plusieurs procédés peuvent être employés :

I ${ }^{\circ}$ Le séchage à l'air : ce procédé est lent et, pour une émulsion épaisse de $100 \mu$, il faut $\mathrm{I}$ à 2 h suivant le degré hygrométrique de l'air ambiant et son agitation; il faut bien davantage pour des émulsions plus épaisses;

$2^{\circ}$ Le séchage sous vide : ce procédé est plus rapide, une émulsion épaisse de $100 \mu$ sèche en io à $\mathrm{I} 5 \mathrm{mn}$;

$3^{0}$ Le séchage par les liquides hydrophiles (alcool, acétone, dioxane, etc.). L'émulsion humide est plongée dans le liquide, on constate qu'elle sèche plus rapidement encore que dans le procédé sous vide.

Séchée à l'air ou au vide, l'émulsion retrouve son état initial (sous réserve qu'une partic de la glycérine incorporée comme plastifiant s'est dissoute et a été perdue pendant l'immersion dans la solution aqueuse d'imprégnation).

Au contraire, nous avons constaté que le séchage par les liquides hydrophiles ne restitue pas à l'émulsion son état initial.

A. Dimensions des traces danș les émulsions traitées par les liquides hydrophiles. - Pensant qu'un tel procédé n'avait pas d'influence spéciale, nous avions utilisé l'acét one pour accélérer le séchage d'une série de plaques imprégnées que nous observions ensuite, avec un faible grossissement et sans mesure des longueurs des traces, dans des expériences impliquant seulement un dénombrement des "étoiles radioactives " et des traces isolées.

Une de ces plaques fut occasionnellement prêtée à $\mathrm{M}^{\mathrm{me}}$ Joliot-Curie qui l'observe avec un grossissement plus fort et nous signala que les traces horizontales des $\alpha$ de $\operatorname{Ra} C^{\prime}$ mesuraient $65 \mu$ environ (au lieu de $38 \mu$ pour des plaques $C_{2}$ séchées à l'air). Cette remarque de Mme Joliot-Curie est donc à l'origine du présent travail et nous l'en remercions bien vivement.

Nous vérifiâmes qu'un séchage imparfait n'était pas la cause de l'allongement observé. L'influence de l'acétone devant être mise en cause, de nouvelles plaques furent préparées, dans diverses conditions, pour en faire une étude systématique.

Soit une trace $\mathrm{AB}$, observée dans une plaque fixée, soit AH sa composante horizontale (parallèle au verre), soit $\mathrm{HB}$ sa composante verticale (perpendiculaire au verre). Appelons $x$ la mesure de $\mathrm{AH}$, $z$ la mesure de HB, les unités de mesures n'étant pas nécessairement les mêmes dans le sens horizontal et dans le sens vertical.

L'un de nous ( ${ }^{(1)}$ a montré que, prenant un groupe monocinétique de rayons $\alpha$, portant sur un graphique en $x^{2}, z^{2}$, les points figuratifs des différentes traces d'inclinaison quelconque, ces points se groupent au voisinage d'une droite. Il a montré également $\left(^{1}\right)$ que cette droite $D$ est plus facile à tracer si plusieurs points figuratifs voisins sont remplacés par leur centre de gravité.

Trois plaques $\mathrm{C} 2$ ont été plongées $3 \mathrm{o} \mathrm{mn}$ dans une solution d'un sel de radium. La plaque témoin, $\mathrm{A}$, a été séchée à l'air; la plaque $\mathrm{B}$ a été. séchée par immersion pendant 3o $\mathrm{mn}$ dans l'alcool, la plaque $\mathrm{C}$ a été séchée par immersion 3o mn dans l'acétone.

Pendant une semaine, les $\alpha$ monocinétiques du radium ont été émis au sein de ces trois plaques sèches. Ensuite nous avons révélé, fixé, et fait les mesures.

La figure I montre les trois droites D obtenues. On peut faire les remarques suivantes :

I $^{\circ}$ Les trois droites coupent l'axe des $X=x^{2}$ en des points très différents : le séchage par les liquides hydrophiles allonge les dimensions horizontales des traces, l'acétone produisant un allongement plus grand que l'alcool;

$2^{\circ} \mathrm{Par}$ contre, dans la limite de la précision expérimentale, les trois droites $\mathrm{D}$ coupent l'axe

(1) L. Vigneron, J. Physique Rad., 1949, 10, 305. 
$\operatorname{des} Z=z^{2}$ au même point : le traitement par les liquides hydrophiles est donc sans inffuence sur la dimension verticale des traces.

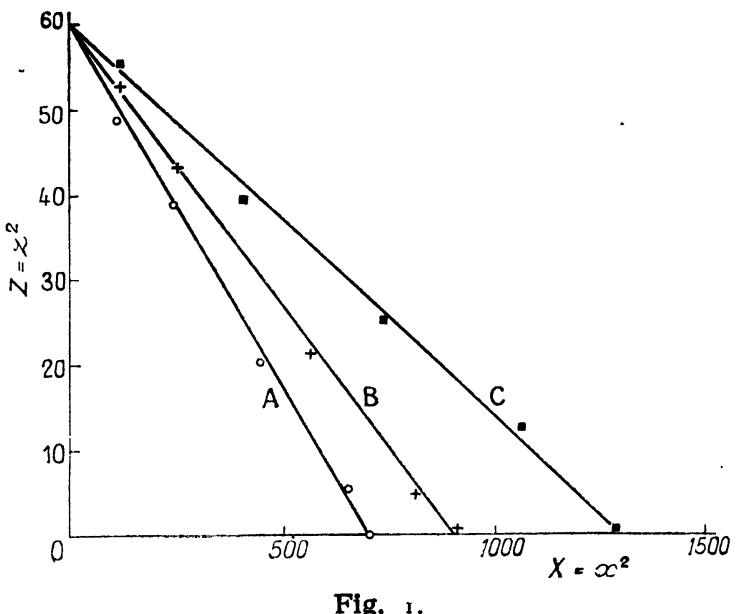

Nous avons également déterminé pour les plaques A, B, C, les histogrammes des parcours des traces horizontales (fig. 2). On peut constater que la dispersion relative des parcours est plus grande dans les plaques traitées par les liquides, ce qui peut être dû à une fluctuation résultant des trous traversés (voir plus loin, § B); il est possible aussi que l'effet de l'acétone ou de l'alcool varie avec la profondeur quond on s'enfonce dans la couche d'émulsion. Nous n'avons pas étudié ce point.

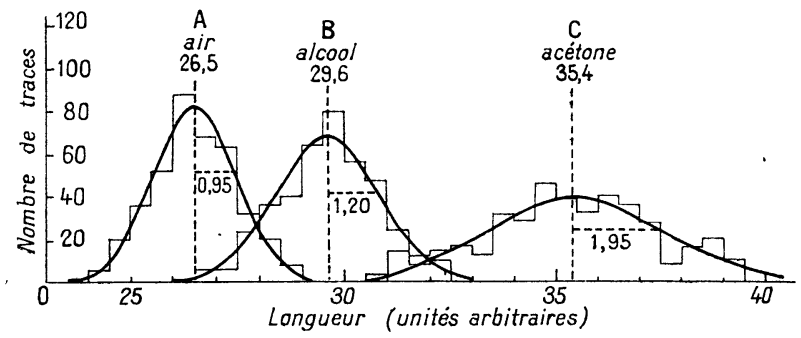

Fig. 2.

L'allongement observé pourrait dépendre également du degré d'hydratation de l'émulsion et de la température du bain déshydratant. Il n'est pas, non plus, nécessairement le même si l'on plonge la plaque à déshydrater directement dans l'alcool ou l'acétone purs ou si on la fait passer sucessivement dans plusieurs bains constitués par des solutions aqueuses d'alcool ou d'acétone, de concentrations croissantes.

L'effet signalé ne nous paraît pas une propriété spéciale de l'alcool ou de l'acétone, nous l'avons noté aussi avec le dioxane. Il faut donc s'attendre à l'observer, avec plus ou moins d'intensité, chaque fois qu'un liquide hydrophile sera utilisé pour déshydrater une émulsion.
Nous n'avons pas étudié systématiquement l'influence des différents facteurs susceptibles de faire varier quantitativement l'effet d'allongement observé sur les traces nucléaires. Toutefois, qualitativement, on observe toujours les phénomènes que nous décrivons.

Nous les signalons donc pour éviter les erreurs qui pourraient être dues à l'emploi inconsidéré d'un liquide hydrophile pour accélérer le séchage des émulsions utilisées dans des expériences de physique nucléaire.

B. Interprétation des faits expérimentaux précédents. - Pour interpréter nos résultats, nous avons été amenés à l'explication suivante, schématisée par la figure 3.

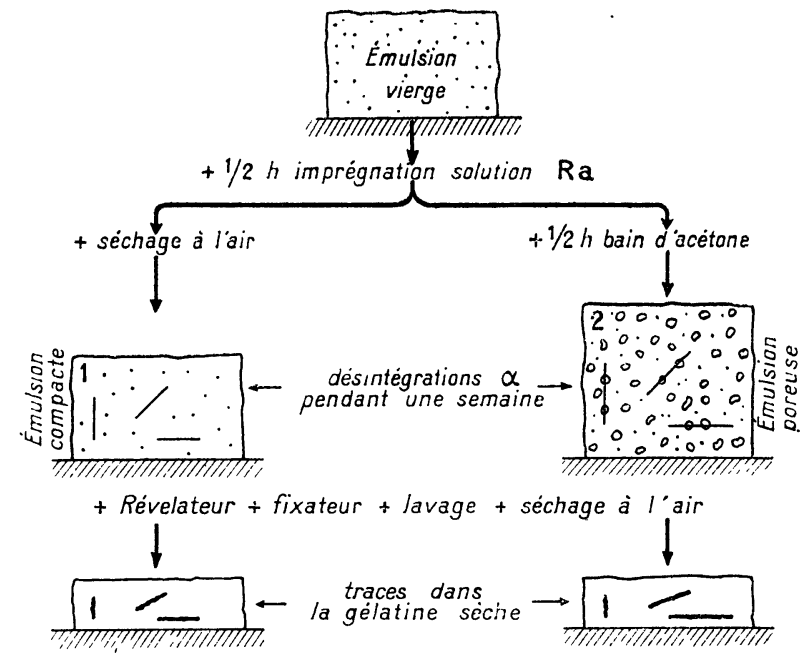

Fig. 3 .

Quand on plonge l'émulsion humide dans le liquide hydrophile, celui-ci la déshydrate mais, de plus, l'émulsion prend une structure en "éponge ". A la sortie du bain déshydratant, le liquide qui remplit les "trous" s'évapore et ceux-ci sont simplement remplis d'air.

Cette structure poreuse reste stable à sec. Elle est immédiatement détruite si l'on mouille à nouveau l'émulsion. Un nouveau séchage à l'air ou au vide aboutit à une érhulsion compacte, sans trous.

A l'examen microscopique, les traces obtenues dans les émulsions déshydratées par les liquides ne se différencient pas notablement des traces obtenues dans les émulsions séchées à l'air. On peut en déduire que la dimension moyenne des trous ne dépasse certainement pas $o, 5 \mu$. Il se peut que la dimension des trous soit bien inférieure à cette limite et soit de l'ordre de grandeur des dimensions intermoléculaires; il s'agirait alors d'un écartement des chaînes formées par les liaisons chimiques dans les macromolécules de la gélatine; nos expériences ne permettent pas de conclure sur ce point. 
Considérons une certaine masse d'émulsion vierge, formée de gélatine, d'halogénures et d'un peu de glycérine; celle-ci part presque totalement à l'imprégnation dans l'eau. Après séchage à l'air, le volume est

$$
V_{1}=V_{g}+V_{h} \text {. }
$$

Considérons une masse égale d'émulsion vierge, mais cette fois déshydratée à l'acétone. Il faut tenir compte du volume, $V_{t}$, des trous, le volume total est alors

$$
V_{2}=V_{g}+V_{h}+V_{l} \text {. }
$$

L'adhérence verre-émulsion impose le maintien des dimensions horizontales. L'émulsion 2, déshydratée, est donc plus épaisse que l'émulsion 1, séchée à l'air ou au vide.

Les coefficients d'affaissement de ces deux émulsions diffèrent : en effet, après révélation, fixage, lavage et séchage final à l'air les deux émulsions ont la même épaisseur, le volume étant dans les deux cas réduit au volume, $V_{g}$, de la gélatine.

Considérons un rayon $\alpha$ dont le parcours est $l_{1}$ s'il se produit au sein de l'émulsion compacte séchée à l'air. L'émulsion poreuse a un pouvoir d'arrêt moindre, car elle est moins dense, à cause des trous. Si le même $\alpha$ s'était produit dans l'émulsion poreuse, comme l'air remplissant les trous à un pouvoir d'arrêt ı 800 fois plus faible que celui de l'émulsion C2, nous pouvons le tenir pour nul, le parcours aurait été plus grand que $l_{1}$, soit

$$
l_{2}=l_{1} \frac{V_{g}+V_{h}+V_{t}}{V_{g}+V_{h}} .
$$

Si les $\alpha$ ont été émis horizontalement, les opérations de développement conservant les dimensions horizontales, la trace dans l'émulsion traitée à l'acétone, a une longueur $l_{2}$ supérieure à la longueur $l_{1}$ de la trace obtenue avec l'émulsion compacte. Nous interprétons donc bien l'allongement expérimentalement observé, pour des traces horizontales, dans le cas des plaques déshydratées par les liquides.

Supposons maintenant que les $\alpha$ aient été émis verticalement. Pour avoir la dimension verticale des traces, il faut tenir compte de la réduction de volume au cours des opérations de développement. Dans le cas de l'émulsion compacte, la trace verticale a comme longueur

$$
l_{1}^{\prime} \doteq l_{1} \frac{V_{g}}{V_{g}+V_{h}} .
$$

Dans le cas de l'émulsion poreuse, la trace verticale a comme longueur

$$
l_{2}^{\prime}=l_{a} \frac{V_{g}}{V_{g}+V_{h}+V_{t}} \text {. }
$$

Mais, un peu plus haut, nous avions exprimé $l_{2}$ en fonction de $l_{1}$. Portant cette valeur dans l'expression ci-dessus, nous avons

$$
l_{2}^{\prime}=l_{1} \frac{V_{g}+V_{h}+V_{t}^{\prime}}{V_{g}+V_{h}} \frac{V_{g}}{V_{g}+V_{h}+I_{l}}=l_{1} \frac{V_{g}}{V_{g}+V_{h}}=l_{1}^{\prime} .
$$

Nous interprétons donc bien l'égalité expérimentalement observée entre les dimensions verticales, $l_{2}^{\prime}$ et $l_{1}^{\prime}$, des traces dans des plaques, indépendamment du procédé employé pour les sécher.

Les traces de rayons $\alpha$ nous ont permis de faire des mesures commodes; le phénomène de porosité que nous avons ainsi expérimentalement mis en évidence existe évidemment pour n'importe quelle émulsion photographique : les émulsions utilisées en Physique nucléaire ne se distinguent, en effet, pas fondamentalement des autres par leur composition.

C. Confirmation directe $d u$ caractère poreux des émulsions traitées à l'acétone. - Par des expériences de pesée, nous avons cherché à confirmer directement les conclusions, concernant la porosité des émulsions, que nous avions déduites des mesures des traces de particules $\alpha$.

I $^{\circ}$ Nous avons pris une plaque llford C2 dont la couche d'émulsion avait une épaisseur voisine de гоo $\mu$. La plaque a été pesée plusieurs fois pendant l'expérience; à la fin de celle-ci, l'émulsion a été enlevée et le verre pesé : nous pouvons donc donner, en milligrammes par centimètre carré, le poids de l'émulsion aux différents moments de l'expérience. Le déroulement de celle-ci est représenté par la figure 4 (à droite de cette figure, la portion CD de la courbe est représentée agrandie).
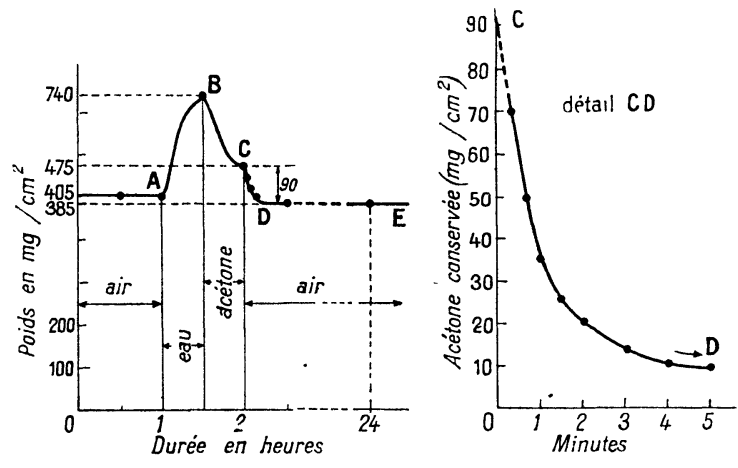

Fig. 4.

Vierge, l'émulsion pèse $405 \mathrm{mg} / \mathrm{cm}^{2}$ [point $\mathrm{A}$, (fig. 4)]. Après un séjour de $30 \mathrm{mn}$ dans l'eau, le poids passe à $740 \mathrm{mg} / \mathrm{cm}^{2}$ (point B). La plaque est alors sortie de l'eau et immédiatement immergée dans un bain déshydratant d'acétone, dans lequel elle séjourne 3o $\mathrm{mn}$; à ce moment, le poids est réduit à $475 \mathrm{mg} / \mathrm{cm}^{2}$ (point $\mathrm{C}$ ). Il reste donc un excès de poịds; la plaque étant à l'air, cet excès disparaît en quelques minutes (portion $\mathrm{CD}$ de la courbe), le poids se fixe à $385 \mathrm{mg} / \mathrm{cm}^{2}$ et ne varie plus.

La différence, $20 \mathrm{mg} / \mathrm{cm}^{2}$, entre le poids au début et à la fin de l'expérience, correspond au départ de la glycérine qui s'est dissoute pendant l'immersion dans l'eau. 
Portons notre attention sur la portion CD. L'excès de poids constaté en $\mathrm{C}$, soit $90 \pm 5 \mathrm{mg} / \mathrm{cm}^{2}$ ne peut être dû à une déshydration imparfaite : l'évaporation, à l'air libre, d'un poids d'eau analogue prendrait plus d'une heure; or l'excès constaté disparaît en quelques minutes. Il ne peut s'agir non plus d'acétone mouillant extérieurement la plaque car, en $\mathrm{C}$, au sortir du bain d'acétone, nous avions pris la précaution d'essuyer la plaque avec un papier buvard fin. La seule interprétation possible de la décroissance $\mathrm{CD}$ est la suivante :

A la sortie du bain d'acétone, en C, l'émulsion conserve dans ses pores $90 \pm 5 \mathrm{mg} / \mathrm{cm}^{2}$ d'acétone, soit à peu près le quart du poids d'émulsion vierge. La grande volatilité de l'acétone explique son évaporation rapide et la disparition de l'excès de poids en quelques minutes.

$2^{\circ}$ Nous avons pris une plaque $\mathrm{C} 2$, , $00 \mu$, identique à la précédente, rendue poreuse par le même traitement (3o $\mathrm{mn}$ dans l'eau suivi de $30 \mathrm{mn}$ dans l'acétone). Après un repos de $24 \mathrm{~h}$, nous l'avons plongée $3 o$ mn dans un bain d'acétone.

Au sortir de ce bain, nous avons constaté que le poids avait augmenté de $35 \pm 5 \mathrm{mg} / \mathrm{cm}^{2}$, cet excès s'évaporant et disparaissant en quelques minutes.
On remarquera que la quantité d'acétone réabsorbable dans les pores est moindre que celle, $90 \pm 5 \mathrm{mg} / \mathrm{cm}^{2}$, qui était présente au sortir du bain de déshydratation; ceci peut être dù à l'une ou l'autre des causes suivantes : resserrement des pores lorsque l'acétone présente en $\mathrm{C}$ s'est évaporée, présence d'air dans les pores formant des bulles gênant une nouvelle pénétration.

Cette expérience montre, de toute façon, qu'une. plaque rendue poreuse par un traitement convenable reste poreuse tant qu'on la garde à sec.

$3^{\circ}$ Il fallait nous assurer que l'acétone ne pénètre pas dans une émulsion compacte, nous avons donc préparé deux plaques témoins, l'une d'émulsion vierge, l'autre privée de sa glycérine par un séjour de 3 o mn dans l'eau suivi par un séchage de $24 \mathrm{~h}$ à l'air libre. Ces deux émulsions compactes ont été immergées pendant $30 \mathrm{mn}$ dans un bain d'acétone. A la sortie de ce bain, aucune variation de poids supérieure à $1 \mathrm{mg} / \mathrm{cm}^{2}$ n'a pu être constatée.

Ces trois expériences de pesée nous paraissent confirmer pleinement le caractère poreux des émulsions déshydratées par les liquides hydrophiles.

Manuscrit reçu le 4 août 1954 . 